\title{
11. ORGANIC GEOCHEMISTRY DSDP LEG 40, CONTINENTAL RISE OF SOUTHWEST AFRICA
}

\author{
James B. Foresman, Phillips Petroleum Company, Bartlesville, Oklahoma
}

\section{INTRODUCTION}

During the course of Leg 40 , the author served as shipboard geologist-geochemist with the responsibility of detecting evidence of petroleum genesis and making recommendations to the Operations Manager and Chief Scientists on whether it was necessary to suspend drilling to avoid penetrating a petroleum or natural gas accumulation and causing pollution. The shipboard methods used to accomplish this function and measurements and observations during the cruise are discussed in the Introduction and Site Reports of this volume. This subsequent section is based on shorebased MS-GLC analyses and characterization of both solid and gas samples collected during the leg. The primary objective of this study was to determine and describe the degree of thermal carbon diagenesis found in strata recovered on Leg 40.

\section{ANALYTICAL METHODS}

Gas was collected in vacuum cans from every core that showed evidence of pressure. Soon after receipt of these samples in our laboratory, their composition was determined by a gas chromatograph equipped with a hydrogen flame ionization detector. This unit permits detection of about $10^{-6}$ the amount which could be determined with the shipboard gas chromatograph which used a matched pair of thermisters for the detector.

A Perkin-Elmer 900 gas chromatograph was used for the laboratory analysis. The valve on the vacuum can was connected to an evacuated Perkin-Elmer gas sampling valve fitted with a 1-cc loop. The sample was injected into a 3.8 meter $\times 3 \mathrm{~mm} 8-100$ mesh Chromosorb 102 column and programmed from $-70^{\circ} \mathrm{C}$ to $100^{\circ} \mathrm{C}$ at a flow rate of $40 \mathrm{ml} / \mathrm{min}$ of helium. The effluent was monitored with a hot wire and hydrogen flame ionization detector that were arranged in series. Nitrogen, oxygen, argon, methane, and carbon dioxide were detected with the hot wire detector and the higher hydrocarbon were detected with the more sensitive flame ionization detector. This technique also permits detection of butenes and pentenes although these were not present.

Carbon isotopic compositions were obtained on a Nier-type $35-\mathrm{cm}$ radius, 90 -degree sector isotope ratio mass spectrometer. Isotope measurements were made on the $\mathrm{m} / \mathrm{e}-45$ and $\mathrm{m} / \mathrm{e}-44$ ions using carbon dioxide as the working gas. Values are reported with the conventional per mil deviations $(\delta)$ from the $\mathrm{C}^{13} / \mathrm{C}^{12}$ ratio of the belemnite (Belemnitella americana) from the Peedee Formation (PDB) of Cretaceous age. Instrument precision is \pm 0.05 per mil.
The 20-cc sediment samples were collected according to standard shipboard sampling procedures using care to minimize contamination while obtaining samples representative of the section penetrated. Carbonate and organic carbon contents were determined by combustion-gas chromatography. A weighted portion of finely ground sample was placed in a special quartz reaction-combustion tube and digested in dilute hydrochloric acid. The evolved carbonate-carbon dioxide was swept by a stream of helium through the combustion tube and water trap and collected in a liquid nitrogen-cooled trap. The carbon dioxide was then volatilized into a gas chromatograph for measurement.

The residual organic carbon was heated in a stream of helium and oxygen to oxidize it to carbon dioxide, which was collected and measured in the same manner as the carbonate carbon dioxide. A heated combustion tube packing assured complete oxidation of organic matter and removal of excess oxygen. Organic carbon samples were combusted to carbon dioxide for mass spectrometry in a vacuum system similar to that described by Craig (1953). Oxidation was carried out at $925^{\circ} \mathrm{C}$ over a copper catalyst in an oxygen atmosphere. Sulfur oxides were removed by a hot manganese dioxide catalyst. Carbon dioxide was purified of water by differential freezing at $-123^{\circ} \mathrm{C}$. Gas components were separated by liquid chromatography prior to conversion to carbon dioxide.

Figure 1 is an index map showing the geography and general bathymetry of the sites drilled on Leg 40 . Data resulting from this study are provided in Tables 1 through 4 and Figures 2 through 6 . The procedure followed for further geochemical characterization of these samples is shown in Figure 2. The solvents shown on this flow chart were distilled in glass prior to use. Carbonate content, organic carbon values, and isotopic data along with sampling depth and stratigraphic age of the sample are provided in Table 1. These data for samples with sufficient organic material to permit more analyses including quantity of lipid material, and values of average odd-even carbon predominance (OEP) are provided in Table 2. Carbon isotopic values for the aromatic and asphaltic fractions of the sediment samples are also provided in Table 2. A tabulation of the relative concentrations expressed as normalized weight percent of $n$-alkane by carbon number over the range that could be analyzed for the individual samples is presented in Table 3. Compositional analysis and $\delta C^{13}$ PDB values for the methane component of the canned gas samples are provided in Table 4 along with sampling depth and sedimentary age. Plots showing the normalized distribution of $n$-alkanes by carbon number 


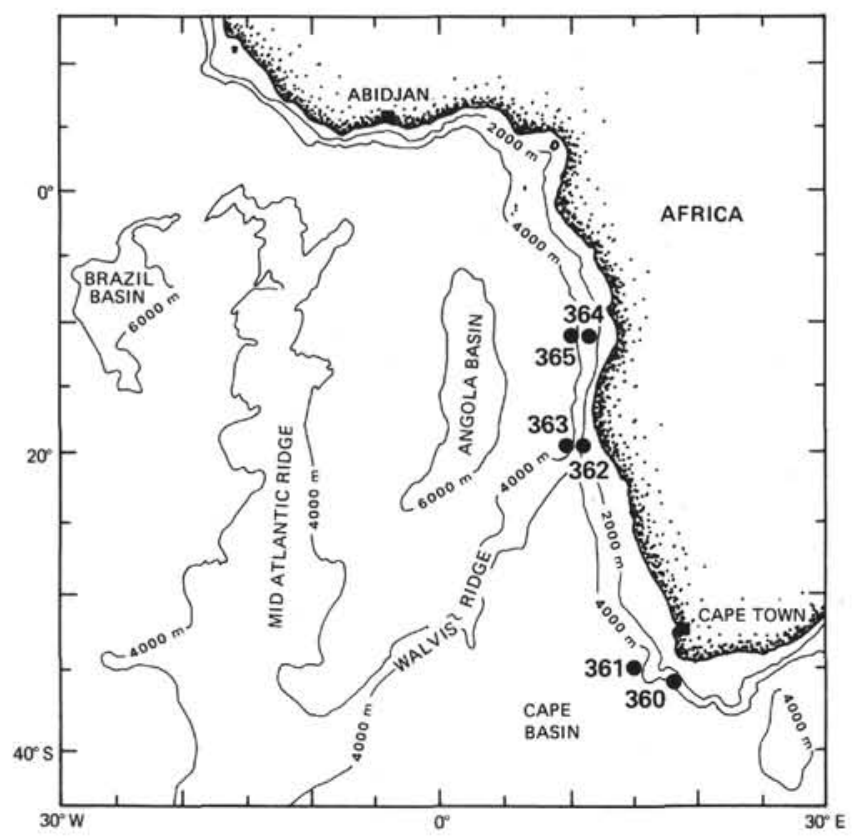

Figure 1. Leg 40 drill sites.

for the carbonaceous samples of Cretaceous age are provided in Figures 3 through 6.

The average Odd-Even Predominance which was developed in this laboratory by Scalan and Smith (1970) is a mathematical expression of the predominance of odd and even carbon numbers as a function of carbon number for the $n$-alkane series. This value is computed according to the following equation:

$$
\mathrm{OEP}=\left[\frac{C_{i}+6 C_{i+2}+C_{i+4}}{4 C_{i+1}+4 C_{i+3}}\right]^{(-1)^{i+1}}
$$

As with Carbon Preference Index (CPI) of Bray and Evans (1961), an OEP value greater than unity indicates an odd number predominance while a value less than unity indicates an even numbered predominance. With increasing depth of burial organic diagenesis generally increases and OEP and CPI values show less of this preference and approach unity. Most crude oils have an OEP value close to unity. Erdman (1975a, b) recently has discussed some of the factors affecting organic diagenesis and the use of OEP as an index for the genesis of petroleum.

\section{RESULTS AND DISCUSSION}

\section{Cape Basin}

Site 360 is located midway down the continental rise south of the Cape of Good Hope. Based on seismic profiling the site was chosen to penetrate a representative Tertiary section. A single hole was drilled to 840 meters subbottom depth, which penetrated a section predominantly consisting of biogenic oozes, chalks, and marly chalks. Coring commenced at 80 meters subbottom in unconsolidated beds of lower Pliocene age. Except for a possible hiatus in the upper
Oligocene, the stratigraphic section is considered to be continuous to total depth in beds of lower Eocene age.

As shown in Table 1, the representative samples from each major lithologic sequence are low in content of organic carbon and generally high in percent of carbonate. The percent of soluble carbon (lipid) is usually less than 0.004 . The isotopic value of the total organic carbon extract is typical of that expected in an open marine environment.

Site 361 was chosen in order to take advantage of an erosional unconformity and to core a section equivalent to the one which underlies the Tertiary sediments at Site 360. A single hole was drilled to 1314 meters subbottom depth. As a result, the cumulative cores from these two sites represent a nearly continuous section over 2000 meters thick with an overlap of only about 100 meters. Drilling at Site 361 penetrated sediments ranging in age from upper Eocene down into beds of the lowermost Aptian or uppermost Barremian Stages of Lower Cretaceous age.

Much of the Lower Cretaceous consists of mudstones, sandy mudstones, and shale that is relatively high in organic content-partly because of the introduction and preservation of terrigenous material. During late Cretaceous and Eocene time biogenic matter and clays were about equal in abundance, producing marly chalks. By the end of the Eocene, however, the terrigenous supply greatly diminished and all younger sediments became pelagic (nanno chalk oozes). The relationship of carbonate carbon versus organic carbon with age is reflected in the values listed in Table 1. The organic carbon content reaches a maximum in the early Cretaceous, averaging about $3 \%$, with the amount of soluble carbon ranging from $0.0012 \%$ to $0.1960 \%$.

As described in the Site Report, the first appearance of gas at Site 361 was noted in Core 28 which was cut from strata of Lower Cretaceous age at a subsea depth beginning at 1000.5 meters. Gas, which caused faint to mild effervescence appeared in every core recovered in the subsequent 314-meter interval. The presence of an ethane component resulted in methane/ethane ratios between 250 and 600 but with no depth trend.

Using the shore-based chromatograph described above, small quantities of propane were found in the gas, and both n-butane and i-butane were detected in the deepest sample. These latter two components, however, occur in disproportionate amounts of 0.001 and 0.0007 weight percent, respectively. This suggests an early stage in hydrocarbon genesis, since the ratio of n-butane to i-butane is less than unity in crude oil.

Carbon isotopic composition for the methane ranges between -66.1 and -55.2 (PDB scale), with no clear trend developing over the interval containing the gas. These values suggest a petroleum gas in the early stage of development that is unlikely to be associated with liquid petroleum. Such light isotopic values for methane are sometimes attributed to bacterial origin (Rosenfeld and Silverman, 1959). Marsh gas, for example, usually has an isotopic value of less than -55 (more negative) while "wet" gas is heavier (more positive) than -50 and often between -30 and -40 . Nonetheless, a theoretical study of the chemical kinetic effects on carbon isotopic compositions by Smith et al. 
TABLE 1

Organic Carbon and Carbonate Carbon Geochemical Data Site 356, Leg 39 and Sites 360 Through 365, Leg 40

\begin{tabular}{|c|c|c|c|c|c|c|c|}
\hline $\begin{array}{c}\text { Sample } \\
\text { (Interval in } \mathrm{cm} \text { ) }\end{array}$ & Age & Lithology & $\begin{array}{c}\text { Percent } \\
\text { Carbonate } \\
\text { Carbon }\end{array}$ & $\begin{array}{l}\text { Total } \\
\text { Percent } \\
\text { Organic } \\
\text { Carbon }\end{array}$ & $\begin{array}{l}\text { Percent } \\
\text { Soluble } \\
\text { Carbon } \\
\text { (Lipid) }\end{array}$ & $\begin{array}{l}\text { Percent } \\
\text { Soluble } \\
\text { (Lipid) } \\
\text { To Total } \\
\text { Organic } \\
\text { Carbon }\end{array}$ & $\begin{array}{c}\delta C^{13} \\
\text { PDB } \\
\text { Extract }\end{array}$ \\
\hline \multicolumn{8}{|l|}{ Site 356} \\
\hline $\begin{array}{l}40-4,12-16 \\
40-4,137-140 \\
40-5,128-132 \\
41-2,12-16 \\
41-2,45-49 \\
41-2,113-117 \\
41-3,12-15 \\
41-3,75-78 \\
41-4,32-36 \\
41-4,92-95\end{array}$ & $\begin{array}{l}\text { Middle Cretaceous } \\
\text { Lower Cretaceous }\end{array}$ & $\begin{array}{l}\text { Mudstone } \\
\text { Marly chalk }\end{array}$ & $\begin{array}{l}0.13 \\
0.20 \\
0.11 \\
0.97 \\
0.11 \\
0.97 \\
2.43 \\
0.81 \\
2.50 \\
6.02\end{array}$ & $\begin{array}{r}0.07 \\
0.05 \\
2.54 \\
2.16 \\
2.54 \\
2.16 \\
9.93 \\
0.68 \\
12.61 \\
0.11\end{array}$ & $\begin{array}{l}0.0086 \\
0.0030 \\
0.0172 \\
0.0031 \\
0.0288 \\
0.0247 \\
0.1564 \\
0.0100 \\
0.2433 \\
0.0074\end{array}$ & $\begin{array}{r}12.3 \\
6.0 \\
0.7 \\
0.1 \\
1.1 \\
1.1 \\
1.6 \\
1.5 \\
1.9 \\
6.7\end{array}$ & $\begin{array}{l}-25.7 \\
-25.8 \\
-27.4 \\
\text { Too small } \\
-27.2 \\
-26.0 \\
-26.1 \\
-23.6 \\
-25.6 \\
-25.7\end{array}$ \\
\hline \multicolumn{8}{|l|}{ Site 360} \\
\hline $\begin{array}{l}1-5,70-74 \\
5-1,40-44 \\
7-1,108-112 \\
10-1,50-64 \\
11-4,69-73 \\
15-2,62-65 \\
18-1,90-94 \\
22-6,82-88 \\
26-2,110-114 \\
35-3,38-41 \\
42-1,72-78 \\
47-2,80-84\end{array}$ & $\begin{array}{l}\text { Middle Miocene } \\
\text { Lower Miocene } \\
\text { Upper Eocene } \\
\text { Middle Eocene }\end{array}$ & $\begin{array}{l}\text { Nannofossil ooze } \\
\text { Nannofossil chalk }\end{array}$ & $\begin{array}{l}9.11 \\
9.18 \\
7.13 \\
9.41 \\
8.48 \\
7.62 \\
7.88 \\
7.28 \\
8.58 \\
6.12 \\
4.38 \\
2.27\end{array}$ & $\begin{array}{l}0.69 \\
0.49 \\
2.16 \\
0.22 \\
0.66 \\
0.06 \\
1.54 \\
0.31 \\
0.24 \\
0.14 \\
0.13 \\
0.67\end{array}$ & $\begin{array}{l}0.0041 \\
0.0084 \\
0.0032 \\
0.0025 \\
0.0040 \\
0.0040 \\
0.0042 \\
0.0017 \\
0.0028 \\
0.0025 \\
0.0019 \\
0.0077\end{array}$ & $\begin{array}{c}0.6 \\
1.7 \\
0.1 \\
1.1 \\
0.6 \\
6.7 \\
0.3 \\
0.6 \\
1.2 \\
1.8 \\
1.5 \\
1.1\end{array}$ & $\begin{array}{l}-23.8 \\
-25.8 \\
-24.8 \\
\text { Too small } \\
-26.5 \\
-27.4 \\
\text { Too small } \\
\text { Too small } \\
-26.6 \\
\text { Too small } \\
-25.3 \\
-23.6\end{array}$ \\
\hline \multicolumn{8}{|l|}{ Site 361} \\
\hline $\begin{array}{l}3-6,83-85 \\
9-5,69-71 \\
11-5,19-22 \\
14-2,65-62 \\
16-1,59-61 \\
17-2,30-33 \\
19-3,43-47 \\
20-2,8-10 \\
21-2,88-92 \\
22-4,131-136 \\
23-4,28-33 \\
24-3,31-36 \\
25-5,36-39 \\
26-6,25-30 \\
27-2,108-113 \\
28-4,31-34 \\
29-4,39-42 \\
29-5,16-20 \\
30-1,76-81 \\
30-3,100-110 \\
31-2,86-91 \\
31-3,6-10 \\
32-1,115-121 \\
32-6,40-44 \\
33-2,54-56 \\
33-2,56-58 \\
34-2,113-119 \\
35-2,142-147 \\
36-1,130-133 \\
37-2,3-8 \\
38-3,12-16 \\
39-3,54-58 \\
41-2,103-108 \\
42-6,107-110\end{array}$ & $\begin{array}{l}\text { Middle Eocene } \\
\text { Upper Paleocene } \\
\text { Upper Cretaceous }\end{array}$ & $\begin{array}{l}\text { Nannofossil mudstone } \\
\text { Shale } \\
\text { Sandy mudstone } \\
\text { Shale } \\
\text { Sandy mudstone } \\
\text { Shale } \\
\text { Mudstone } \\
\text { Shale } \\
\\
\text { Mudstone } \\
\text { Sandy mudstone } \\
\text { Shale } \\
\text { Sandy mudstone } \\
\text { Shale } \\
\text { Sandstone } \\
\text { Shale } \\
\text { Mudstone }\end{array}$ & $\begin{array}{l}0.06 \\
0.03 \\
0.04 \\
0.01 \\
0.02 \\
0.01 \\
0.04 \\
0.04 \\
0.04 \\
0.04 \\
0.04 \\
0.06 \\
0.08 \\
0.79 \\
0.12 \\
0.08 \\
0.04 \\
0.13 \\
0.03 \\
0.02 \\
0.01 \\
0.04 \\
0.92 \\
0.90 \\
0.29 \\
0.28 \\
0.03 \\
0.06 \\
0.13 \\
0.05 \\
0.03 \\
0.02 \\
0.67 \\
0.34\end{array}$ & $\begin{array}{r}0.04 \\
0.10 \\
0.04 \\
0.13 \\
0.24 \\
0.27 \\
0.38 \\
0.98 \\
0.26 \\
0.13 \\
0.17 \\
0.41 \\
0.04 \\
0.05 \\
0.24 \\
0.74 \\
4.08 \\
6.24 \\
4.75 \\
3.80 \\
2.97 \\
3.21 \\
0.81 \\
5.88 \\
2.55 \\
2.32 \\
2.30 \\
0.67 \\
3.07 \\
13.89 \\
3.71 \\
0.95 \\
2.75 \\
1.89\end{array}$ & $\begin{array}{l}0.0034 \\
0.0371 \\
0.0090 \\
0.0052 \\
0.0047 \\
0.0047 \\
0.0053 \\
0.0260 \\
0.0029 \\
0.0019 \\
0.0030 \\
0.0053 \\
0.0036 \\
0.0019 \\
0.0012 \\
0.0566 \\
0.1484 \\
0.1817 \\
0.1545 \\
0.0787 \\
0.0258 \\
0.0415 \\
0.0216 \\
0.1960 \\
0.0559 \\
0.0590 \\
0.1120 \\
0.0610 \\
0.0663 \\
0.1680 \\
0.0545 \\
0.0454 \\
0.0488 \\
0.0280\end{array}$ & $\begin{array}{r}8.5 \\
37.1 \\
22.5 \\
4.0 \\
2.0 \\
1.7 \\
1.4 \\
2.6 \\
1.1 \\
1.5 \\
1.8 \\
1.3 \\
9.0 \\
3.8 \\
0.5 \\
7.6 \\
3.6 \\
2.9 \\
3.2 \\
2.1 \\
3.2 \\
1.3 \\
2.7 \\
3.3 \\
2.2 \\
2.5 \\
4.9 \\
9.1 \\
2.2 \\
1.2 \\
1.5 \\
4.8 \\
1.8 \\
1.5\end{array}$ & $\begin{array}{l}-24.6 \\
-27.8 \\
-24.8 \\
-26.9 \\
-26.0 \\
-25.6 \\
-26.8 \\
-26.3 \\
\text { Too small } \\
-26.3 \\
-26.6 \\
-25.9 \\
-26.1 \\
-25.7 \\
-25.8 \\
-27.2 \\
-26.8 \\
-28.3 \\
-27.7 \\
-26.5 \\
-25.0 \\
-25.7 \\
-26.1 \\
-28.7 \\
-28.6 \\
-28.4 \\
-26.7 \\
-25.3 \\
-26.4 \\
-25.3 \\
-26.0 \\
-26.4 \\
-26.9 \\
-25.8 \\
\end{array}$ \\
\hline
\end{tabular}


TABLE 1 - Continued

\begin{tabular}{|c|c|c|c|c|c|c|c|}
\hline $\begin{array}{c}\text { Sample } \\
\text { (Interval in } \mathrm{cm} \text { ) }\end{array}$ & Age & Lithology & $\begin{array}{c}\text { Percent } \\
\text { Carbonate } \\
\text { Carbon }\end{array}$ & $\begin{array}{l}\text { Total } \\
\text { Percent } \\
\text { Organic } \\
\text { Carbon }\end{array}$ & $\begin{array}{l}\text { Percent } \\
\text { Soluble } \\
\text { Carbon } \\
\text { (Lipid) }\end{array}$ & $\begin{array}{l}\text { Percent } \\
\text { Soluble } \\
\text { (Lipid) } \\
\text { To Total } \\
\text { Organic } \\
\text { Carbon }\end{array}$ & $\begin{array}{l}\delta \mathrm{C}_{\mathrm{PDB}}^{13} \\
\text { Extract }\end{array}$ \\
\hline $\begin{array}{l}43-2,119-123 \\
43-4,11-15 \\
45-3,25-31 \\
46-3,140-145 \\
47-1,72-77 \\
48-2,30-35\end{array}$ & & $\begin{array}{l}\text { Sandy mudstone } \\
\text { Shale } \\
\text { Sandy mudstone } \\
\text { Sandy mudstone }\end{array}$ & $\begin{array}{l}0.03 \\
0.01 \\
0.32 \\
0.26 \\
0.04 \\
0.09\end{array}$ & $\begin{array}{l}1.37 \\
2.94 \\
3.74 \\
3.22 \\
4.35 \\
3.12\end{array}$ & $\begin{array}{l}0.0373 \\
0.0116 \\
0.0460 \\
0.1824 \\
0.1496 \\
0.0086\end{array}$ & $\begin{array}{l}2.7 \\
0.4 \\
1.2 \\
5.7 \\
3.4 \\
0.3\end{array}$ & $\begin{array}{l}-25.5 \\
-26.1 \\
-27.0 \\
-26.6 \\
-25.9 \\
-24.0\end{array}$ \\
\hline \multicolumn{8}{|l|}{ Hole 362} \\
\hline $\begin{array}{l}1-3,26-30 \\
7-3,26-30 \\
10-3,26-30 \\
12-2,66-70 \\
14-5,105-109 \\
16-2,89-91 \\
18-1,66-70 \\
20-1,105-110 \\
22-2,64-68 \\
24-1,88-92 \\
26-1,68-72 \\
29-3,51-54 \\
31-2,90-94 \\
33-6,39-42 \\
35-1,27-29 \\
38-4,51-54 \\
40-1,45-49\end{array}$ & $\begin{array}{l}\text { Lower Pliocene } \\
\text { Upper Miocene }\end{array}$ & $\begin{array}{l}\text { Marly calc nanno ooze } \\
\text { Marly nanno chalk } \\
\text { Nanno chalk } \\
\text { Marly nanno chalk }\end{array}$ & $\begin{array}{l}7.58 \\
4.30 \\
6.72 \\
5.34 \\
5.90 \\
6.67 \\
5.35 \\
8.05 \\
8.12 \\
9.56 \\
7.36 \\
7.60 \\
7.64 \\
4.97 \\
7.71 \\
6.00 \\
7.02\end{array}$ & $\begin{array}{l}2.66 \\
3.58 \\
4.20 \\
2.37 \\
2.06 \\
1.59 \\
0.63 \\
0.56 \\
0.72 \\
0.55 \\
0.65 \\
0.06 \\
0.04 \\
0.27 \\
0.41 \\
0.62 \\
0.13\end{array}$ & $\begin{array}{l}0.2739 \\
0.0476 \\
0.0188 \\
0.0325 \\
0.0301 \\
0.0174 \\
0.0130 \\
0.0096 \\
0.0117 \\
0.0089 \\
0.0133 \\
0.0077 \\
0.0212 \\
0.0080 \\
0.0043 \\
0.0024 \\
0.0021\end{array}$ & $\begin{array}{r}10.3 \\
1.3 \\
0.4 \\
1.4 \\
1.5 \\
1.1 \\
2.1 \\
1.7 \\
1.6 \\
1.6 \\
2.0 \\
12.8 \\
53.0 \\
3.0 \\
1.0 \\
0.4 \\
1.6\end{array}$ & $\begin{array}{l}-27.9 \\
-23.6 \\
-24.8 \\
-22.4 \\
-23.7 \\
-22.7 \\
-22.2 \\
-24.8 \\
-24.7 \\
-25.7 \\
-24.5 \\
-25.4 \\
-25.5 \\
-25.2 \\
-26.6 \\
-26.9 \\
-26.2\end{array}$ \\
\hline \multicolumn{8}{|l|}{ Hole 362A } \\
\hline $\begin{array}{l}2-2 \\
4-1 \\
5-1 \\
7-1 \\
9-2\end{array}$ & $\begin{array}{l}\text { Middle Oligocene } \\
\text { Lower Oligocene } \\
\text { Upper Eocene } \\
\text { Middle Eocene }\end{array}$ & Marly nanno chalk & $\begin{array}{r}4.55 \\
9.11 \\
10.66 \\
8.14 \\
8.06\end{array}$ & $\begin{array}{l}0.13 \\
0.10 \\
0.06 \\
0.08 \\
0.07\end{array}$ & $\begin{array}{l}0.0034 \\
0.0026 \\
0.0041 \\
0.0050 \\
0.0047\end{array}$ & $\begin{array}{l}2.6 \\
2.6 \\
6.8 \\
6.2 \\
6.7\end{array}$ & $\begin{array}{l}-26.3 \\
-24.4 \\
-25.5 \\
-25.5 \\
-25.6\end{array}$ \\
\hline Site 363 & & & & & & & \\
\hline $\begin{array}{l}18-2,73-77 \\
20-1,68-72 \\
23-1,85-89 \\
26-1,85-89 \\
26-4,50-54 \\
26-4 \\
28-2,12-16 \\
30-2,12-16 \\
32-5,22-26 \\
34-1,118-122 \\
36-3,113-117 \\
37-5,78-82 \\
39-2,98-102\end{array}$ & Upper Cretaceous & $\begin{array}{l}\text { Nannofossil chalk } \\
\text { Marly nanno chalk } \\
\text { Marly limestone } \\
\text { Limestone } \\
\text { Marly limestone } \\
\text { Limestone }\end{array}$ & $\begin{array}{r}10.36 \\
3.69 \\
3.25 \\
9.80 \\
6.43 \\
10.24 \\
3.93 \\
3.14 \\
2.43 \\
9.16 \\
6.48 \\
9.95 \\
10.36\end{array}$ & $\begin{array}{l}0.69 \\
1.08 \\
0.97 \\
0.04 \\
0.07 \\
0.19 \\
0.19 \\
0.82 \\
0.08 \\
0.18 \\
0.17 \\
0.09 \\
0.14\end{array}$ & $\begin{array}{l}0.0047 \\
0.0044 \\
0.0020 \\
0.0246 \\
0.0342 \\
0.0188 \\
0.0037 \\
0.0027 \\
0.0018 \\
0.0017 \\
0.0040 \\
0.0020 \\
0.0048\end{array}$ & $\begin{array}{r}0.7 \\
0.4 \\
0.2 \\
2.4 \\
48.8 \\
9.9 \\
1.9 \\
0.3 \\
2.2 \\
0.9 \\
2.4 \\
2.2 \\
3.4\end{array}$ & $\begin{array}{l}-25.9 \\
-26.5 \\
-26.2 \\
-26.1 \\
-27.4 \\
-27.8 \\
-25.7 \\
-26.7 \\
-26.5 \\
-26.2 \\
-27.5 \\
-26.9 \\
-26.6\end{array}$ \\
\hline \multicolumn{8}{|l|}{ Site 364} \\
\hline $\begin{array}{l}1-2,18-22 \\
3-1,40-44 \\
5-2,89-93 \\
7-1,11-15 \\
8-1,88-92 \\
10-6,37-40 \\
13-2,66-69 \\
15-2,65-69 \\
17-1,14-18 \\
20-3,120-122 \\
21-4,100-102 \\
22-4,62-24 \\
23-1,117-120 \\
23-1 \\
24-2,27-30\end{array}$ & $\begin{array}{l}\text { Pleistocene } \\
\text { Pliocene } \\
\text { Oligocene } \\
\text { Eocene } \\
\text { Paleocene } \\
\text { Upper Cretaceous }\end{array}$ & $\begin{array}{l}\text { Calc mudstone } \\
\text { Calc nanno ooze } \\
\text { Marly nanno ooze } \\
\text { Pelagic clay } \\
\text { Nannofossil chalk }\end{array}$ & $\begin{array}{l}1.43 \\
4.56 \\
1.45 \\
0.07 \\
2.38 \\
8.46 \\
8.86 \\
8.68 \\
8.94 \\
1.36 \\
0.12 \\
0.04 \\
3.08 \\
0.07 \\
0.22\end{array}$ & $\begin{array}{r}0.17 \\
0.38 \\
0.11 \\
0.06 \\
0.08 \\
0.07 \\
0.07 \\
0.04 \\
0.05 \\
12.80 \\
13.60 \\
0.07 \\
17.59 \\
15.66 \\
6.76\end{array}$ & $\begin{array}{l}0.0046 \\
0.0081 \\
0.0042 \\
0.0050 \\
0.0100 \\
0.0087 \\
0.0088 \\
0.0058 \\
0.0046 \\
0.1549 \\
0.3454 \\
0.0048 \\
0.6907 \\
0.3732 \\
0.1070\end{array}$ & $\begin{array}{r}2.7 \\
2.1 \\
3.8 \\
8.3 \\
12.5 \\
12.4 \\
12.6 \\
14.5 \\
9.2 \\
1.2 \\
2.5 \\
6.8 \\
3.9 \\
2.4 \\
1.5\end{array}$ & $\begin{array}{l}-25.6 \\
\text { Too small } \\
-27.0 \\
-26.7 \\
-25.4 \\
-27.8 \\
-25.8 \\
-25.4 \\
\text { Too small } \\
-26.5 \\
-26.5 \\
-27.3 \\
-25.2 \\
-27.9 \\
-28.6\end{array}$ \\
\hline
\end{tabular}


TABLE 1 - Continued

\begin{tabular}{|c|c|c|c|c|c|c|c|}
\hline $\begin{array}{c}\text { Sample } \\
\text { (Interval in } \mathrm{cm} \text { ) }\end{array}$ & Age & Lithology & $\begin{array}{l}\text { Percent } \\
\text { Carbonate } \\
\text { Carbon }\end{array}$ & $\begin{array}{c}\text { Total } \\
\text { Organic } \\
\text { Carbon }\end{array}$ & $\begin{array}{l}\text { Percent } \\
\text { Soluble } \\
\text { Carbon } \\
\text { (Lipid) }\end{array}$ & $\begin{array}{c}\text { Percent } \\
\text { Soluble } \\
\text { (Lipid) } \\
\text { To Total } \\
\text { Organic } \\
\text { Carbon }\end{array}$ & $\begin{array}{l}\delta C_{\mathrm{PDB}}^{13} \\
\text { Extract }\end{array}$ \\
\hline $25-6,79-81$ & \multirow{28}{*}{ Lower Cretaceous } & Mudstone & 0.25 & 0.06 & 0.0043 & 7.5 & Too small \\
\hline $26-6,143-145$ & & Marly nanno chalk & 5.41 & 0.07 & 0.0028 & 4.0 & -25.7 \\
\hline $28 \mathrm{CC}$ & & & 8.94 & 0.06 & 0.0010 & 1.7 & -26.8 \\
\hline $29 \mathrm{CC}$ & & Limestone & 9.89 & 0.09 & 0.0022 & 2.4 & -25.6 \\
\hline $30 \mathrm{CC}$ & & & 9.44 & 0.10 & 0.0067 & 6.7 & -27.0 \\
\hline $31-1,27-29$ & & Marly limestone & 2.56 & 1.03 & 0.0188 & 1.8 & -27.3 \\
\hline $32-2,126-128$ & & & 7.92 & 0.61 & 0.0271 & 4.4 & -26.8 \\
\hline $32-3,53-56$ & & & 4.34 & 0.09 & 0.0067 & 7.4 & -26.6 \\
\hline $33-3,72-74$ & & Limestone & 3.19 & 1.01 & 0.0126 & 1.2 & -26.7 \\
\hline $34-1,104-107$ & & & 1.57 & 0.17 & 0.0050 & 2.9 & -26.0 \\
\hline $35-3,70-73$ & & Marly limestone & 6.70 & 1.39 & 0.0294 & 2.1 & -26.7 \\
\hline $37-2,11-14$ & & & 7.39 & 0.36 & 0.0097 & 2.7 & -27.4 \\
\hline $38-2,73-76$ & & & 7.70 & 1.31 & 0.0475 & 3.6 & -28.0 \\
\hline $38-3,132-136$ & & Limestone & 6.13 & 3.51 & 0.0976 & 2.8 & -27.4 \\
\hline $39-1,132-134$ & & Shale & 2.66 & 5.51 & 0.1467 & 2.7 & -27.9 \\
\hline $39-2,123-125$ & & Limestone & 5.10 & 6.22 & 0.1702 & 2.7 & -28.2 \\
\hline $39-3,60-63$ & & & 2.71 & 7.27 & 0.2209 & 3.0 & -28.4 \\
\hline $40-2,120-123$ & & Shale & 4.12 & 3.08 & 0.2133 & 6.9 & -28.2 \\
\hline $40-3,117-120$ & & Limestone & 4.05 & 4.03 & 0.1178 & 2.9 & -28.2 \\
\hline $41-4,53-55$ & & Marly limestone & 4.74 & 5.70 & 0.1474 & 2.6 & -27.6 \\
\hline $42-2,64-66$ & & & 1.68 & 6.72 & 0.2090 & 3.1 & -27.1 \\
\hline $42-3,46-49$ & & Shale & 0.13 & 22.63 & 1.2487 & 5.5 & -26.7 \\
\hline $42-4,85-88$ & & & 4.11 & 10.70 & 0.5302 & 5.0 & -26.8 \\
\hline $43-3,114-117$ & & & 0.05 & 6.70 & 2.8037 & 41.8 & -22.5 \\
\hline $44-2,4-6$ & & Marly limestone & 0.20 & 19.61 & 0.9441 & 4.8 & -24.4 \\
\hline $44-3,24-26$ & & Limestone & 0.22 & 24.00 & 1.6502 & 6.9 & -24.0 \\
\hline $45-3,130-133$ & & Shale & 3.03 & 22.34 & 1.4180 & 6.3 & -24.2 \\
\hline $46-1,146-150$ & & Dolomite & 9.73 & 0.51 & 0.0176 & 3.4 & -26.8 \\
\hline \multicolumn{8}{|l|}{ Site 365} \\
\hline $2-3,56-58$ & Upper Cretaceous & Mudstone & 0.71 & 21.26 & 0.700 & 3.3 & -26.6 \\
\hline $3-1,80-82$ & Lower Cretaceous & Claystone & 0.09 & 17.30 & 0.2730 & 1.6 & -28.7 \\
\hline $3-1,78-80$ & & & 0.04 & 17.23 & 0.2640 & 1.5 & -29.0 \\
\hline $5-2,100-104$ & & Mudstone & 0.05 & 0.86 & 0.0118 & 1.4 & -27.0 \\
\hline $6-1,73-77$ & & & 0.08 & 0,37 & 0.0032 & 0.9 & -27.0 \\
\hline $7-2,85-89$ & & & 0.09 & 0.20 & 0.0028 & 1.4 & -26.5 \\
\hline
\end{tabular}

(1971, p. 21-24) predicts a progressive change in isotopic compositions with depth toward heavier values as organic diagenesis becomes more pronounced. The measured isotopic values for methane in this section are similar to values predicted from this kinetic model. Therefore, this gas is believed to have originated primarily from abiogenic degradation of the organic matter and represent the early products of organic diagenesis.

Most samples from lower Cretaceous strata contain enough hydrocarbons to be classed as source beds of petroleum according to criteria of Phillipi (1956). However, the OEP value indicates that organic diagenesis has not proceeded very far in this interval. The OEP values listed in the last column of Table 2 indicate that the lipid (oil) fraction has a marked odd number carbon predominance that also is evident from the n-alkane plot shown in Figure 4. Moreover, the low amount of lipids relative to kerogen content further suggests an early stage of genesis. In sediments protected from molecular oxygen, the ratio of lipids to total organic matter should increase with age and depth of burial as reactions during the genesis of petroleum progress (Erdman, 1975b). The carbon isotopic values of the lipid fractions reflect the influx of terrigenous material into a marine environment during Lower Cretaceous time.

\section{Walvis Ridge}

Sites $362,362 \mathrm{~A}$, and 363 are located on the Abutment Plateau segment of the Walvis Ridge where it joins the continental margin of southwest Africa. The prominent submarine ridge separates the Cape and Angola basins. Drilling was terminated at a subsea depth of 806 meters at Site 362 and 1081 meters at the offset Hole $362 \mathrm{~A}$. The section penetrated at Holes 362 and $362 \mathrm{~A}$ is continuous in age from Holocene to lower Eocene. It consists of biogenic oozes, both siliceous and calcareous, chalk, marly chalk, and limestone.

A continuous gassy section was penetrated in beds of diatomaceous nanno ooze of Pleistocene and midMiocene age which lie between the sediment-water interface and a depth of 464 meters. The gas consists primarily of methane, generally less than 4 weight percent carbon dioxide, and hydrogen sulfide estimated to occur in proportions of about $25 \mathrm{ppm}$. The isotopic 
TABLE 2

Organic Geochemical Data, Leg 40

\begin{tabular}{|c|c|c|c|c|c|c|c|c|c|c|c|c|c|c|}
\hline \multirow{3}{*}{$\begin{array}{c}\text { Sample } \\
\text { (Interval } \\
\text { in } \mathrm{cm} \text { ) }\end{array}$} & \multirow{3}{*}{$\begin{array}{l}\text { Depth } \\
\text { Below } \\
\text { Mudline }\end{array}$} & \multirow[b]{3}{*}{$\begin{array}{l}\text { Stratigraphic } \\
\text { Age }\end{array}$} & \multirow[b]{3}{*}{$\begin{array}{c}\text { Carbonate }^{\mathrm{a}} \\
\text { Carbon }\end{array}$} & \multirow[b]{3}{*}{$\mathrm{CaCO}_{3}$} & \multicolumn{7}{|c|}{ Weight Percent } & \multirow{2}{*}{\multicolumn{2}{|c|}{$\delta \mathrm{C}_{\mathrm{PDB}}^{13}$}} & \multirow{3}{*}{$\begin{array}{c}\text { Odd- } \\
\text { Even } \\
\text { Predom- } \\
\text { inance } \\
\text { OEP }\end{array}$} \\
\hline & & & & & \multirow{2}{*}{\multicolumn{3}{|c|}{ Organic Carbon ${ }^{\mathbf{a}}$}} & \multicolumn{3}{|c|}{ Lipid Fractions ${ }^{b}$} & \multirow[b]{2}{*}{$\begin{array}{l}\text { Total } \\
\text { Lipids }\end{array}$} & & & \\
\hline & & & & & $\begin{array}{r}\text { Or } \\
\text { Total }\end{array}$ & & & $\begin{array}{l}\text { Satu- } \\
\text { rates }\end{array}$ & $\begin{array}{l}\text { Aro- } \\
\text { matics }\end{array}$ & $\begin{array}{l}\text { Asphal- } \\
\text { tics }\end{array}$ & & $\begin{array}{c}\text { Aro- } \\
\text { matics }\end{array}$ & $\begin{array}{c}\text { Asphal- } \\
\text { tics }\end{array}$ & \\
\hline \multicolumn{15}{|l|}{ Site $356^{d}$} \\
\hline $41-3,12-15$ & & Lower & 2.43 & 20.25 & 9.93 & 0.16 & 9.77 & 5.7 & 21.9 & 72.4 & 1.6 & -30.6 & -25.7 & 1.90 \\
\hline $41-4,32-36$ & & Cretaceous & 2.50 & 20.83 & 12.61 & 0.24 & 12.37 & 2.7 & 23.1 & 74.2 & 1.9 & -32.4 & $-24: 7$ & 2.37 \\
\hline \multicolumn{15}{|l|}{ Site 361} \\
\hline $28-4,39-42$ & 1005.39 & & 0.08 & 0.67 & 0.74 & 0.06 & 0.68 & 25.3 & 46.8 & 27.9 & 8.1 & -25.2 & -27.7 & e \\
\hline $33-2,54-56$ & 1069.04 & & 0.28 & 2.33 & 2.32 & 0.06 & 2.26 & 9.4 & 26.9 & 63.7 & 2.6 & -25.9 & -27.1 & - \\
\hline $34-2,113-119$ & 1079.13 & & 0.03 & 0.25 & 2.30 & 0.11 & 2.19 & 8.0 & 57.0 & 35.0 & 4.8 & -24.9 & -25.5 & 1.95 \\
\hline $35-2,142-147$ & 1088.92 & & 0.06 & 0.50 & 0.67 & 0.06 . & 0.61 & 5.8 & 70.3 & 23.9 & 9.0 & -26.0 & -25.9 & - \\
\hline $36-1,130-133$ & 1096.30 & & 0.13 & 1.08 & 3.07 & 0.07 & 3.00 & 6.0 & 39.9 & 54.1 & 2.3 & -26.2 & -25.8 & 2.80 \\
\hline $37-2,3-8$ & 1106.53 & & 0.04 & 0.33 & 13.50 & 0.17 & 13.33 & 5.6 & 40.1 & 54.4 & 1.3 & -25.9 & -25.9 & 1.51 \\
\hline $38-3,12-16$ & 1117.62 & & 0.03 & 0.25 & 3.71 & 0.05 & 3.66 & 8.9 & 38.1 & 53.0 & 1.3 & -25.7 & -26.2 & 2.89 \\
\hline $39-3,54-58$ & 1127.54 & & 0.02 & 0.17 & 0.95 & 0.04 & 0.91 & 25.7 & 52.2 & 22.1 & 4.2 & -25.1 & -24.9 & - \\
\hline $41-2,103-108$ & 1164.53 & & 0.67 & 5.58 & 2.75 & 0.05 & 2.70 & 18.5 & 18.8 & 62.6 & 1.8 & -25.3 & -25.5 & - \\
\hline 43-2, & 1201.00 & & 0.03 & 0.25 & 1.37 & 0.04 & 1.33 & 9.0 & 74.7 & 16.3 & 2.9 & -24.8 & -25.3 & - \\
\hline $43-4,119-123$ & 1205.09 & & 0.01 & 0.08 & 2.94 & 0.01 & 2.93 & 7.6 & 46.8 & 45.6 & 0.3 & -25.3 & -24.7 & 0.42 \\
\hline $45-3,25-31$ & 1241.25 & & 0.32 & 2.67 & 3.74 & 0.05 & 3.69 & 17.8 & 44.2 & 38.1 & 1.3 & -26.0 & -25.1 & 2.46 \\
\hline $46-3,140-145$ & 1261.40 & & 0.26 & 2.17 & 3.22 & 0.18 & 3.04 & 7.4 & 35.0 & 57.6 & 5.6 & -26.6 & -26.1 & 2.88 \\
\hline $47-1,72-77$ & 1267.22 & & 0.04 & 0.33 & 4.35 & 0.15 & 4.20 & 49.5 & 29.7 & 20.9 & 3.4 & -25.6 & -25.5 & - \\
\hline \multicolumn{15}{|l|}{ Site 364} \\
\hline $20-3,120-122$ & 581.70 & Upper & 1.36 & 11.33 & 13.80 & 0.15 & 13.65 & 5.9 & 21.9 & 72.3 & 1.2 & -27.5 & -30.4 & 3.33 \\
\hline $21-4,100-102$ & 602.00 & Cretaceous & 0.12 & 1.00 & 13.60 & 0.35 & 13.25 & 16.9 & 15.3 & 67.8 & 2.6 & - & -27.2 & 3.14 \\
\hline $23-1,117-120$ & 645.17 & Middle & 3.08 & 25.67 & 17.59 & 0.69 & 16.90 & 7.0 & 25.3 & 67.6 & 3.9 & - & - & 2.46 \\
\hline $23-1,117-120$ & 645.17 & Cretaceous & 0.07 & 0.58 & 15.66 & 0.37 & 15.29 & 10.9 & 24.3 & 64.8 & 2.4 & -28.1 & -27.7 & 2.95 \\
\hline $24-2,27-30$ & 674.27 & & 0.22 & 1.83 & $6: 76$ & 0.11 & 6.65 & 16.3 & 16.1 & 67.6 & 1.6 & -29.2 & -29.0 & 4.15 \\
\hline $39-1,132-134$ & 968.32 & Lower & 2.56 & 22.17 & 5.51 & 0.15 & 5.36 & 13.0 & 31.4 & 55.6 & 2.7 & -30.7 & -28.1 & - \\
\hline $39-2,123-125$ & 969.73 & Cretaceous & 5.67 & 47.25 & 0.62 & 0.17 & 0.45 & 7.5 & 16.6 & 75.9 & 27.4 & -28.5 & -28.3 & - \\
\hline $39-3,60-63$ & 970.80 & & 2.71 & 22.58 & 7.27 & 0.22 & 7.05 & 11.3 & 35.5 & 53.1 & 3.0 & -28.3 & -28.8 & - \\
\hline $40-2,120-123$ & 988.70 & & 4.12 & 34.33 & 3.08 & 0.21 & 2.87 & 7.6 & 21.0 & 71.4 & 6.8 & -28.9 & -28.0 & 1.58 \\
\hline $40-3,117-120$ & 990.17 & & 4.05 & 33.75 & 4.03 & 0.13 & 3.91 & 18.5 & 33.9 & 58.6 & 3.0 & -28.6 & -28.1 & - \\
\hline $41-4,53-55$ & 1010.03 & & 4.74 & 39.50 & 5.70 & 0.15 & 5.55 & 4.6 & 23.6 & 71.8 & 2.6 & -26.4 & -27.2 & 2.58 \\
\hline $42-2,64-66$ & 1026.14 & & 1.68 & 14.00 & 6.72 & 0.21 & 6.51 & 5.3 & 13.9 & 80.8 & 3.1 & -27.1 & -27.2 & 1.70 \\
\hline $42-3,46-49$ & 1027.46 & & 0.13 & 1.08 & 22.68 & 1.25 & 31.43 & 2.6 & 28.9 & 68.5 & 5.5 & -26.4 & -26.6 & 2.25 \\
\hline $42-4,85-88$ & 1029.35 & & 4.11 & 34.25 & 10.70 & 0.53 & 10.17 & 16.6 & 20.8 & 62.7 & 5.0 & -26.4 & -26.7 & - \\
\hline $43-3,114-117$ & 1037.64 & & 0.05 & 0.42 & 6.70 & 2.80 & 3.90 & 0.6 & 31.0 & 68.4 & 41.8 & - & - & 1.83 \\
\hline $44-2,4-6$ & 1044.54 & & 0.20 & 1.64 & 19.61 & 0.94 & 18.67 & 8.1 & 29.9 & 62.1 & 4.8 & -24.9 & -25.0 & - \\
\hline $44-3,24-26$ & 1046.24 & & 0.22 & 1.83 & 24.00 & 1.65 & 22.35 & 2.1 & 15.0 & 82.9 & 6.9 & -25.8 & -25.2 & - \\
\hline $45-2,130-133$ & 1064.80 & & 3.03 & 25.25 & 22.34 & 1.42 & 20.92 & 0.9 & 14.0 & 85.2 & 6.4 & -25.9 & -24.9 & 2.01 \\
\hline \multicolumn{15}{|l|}{ Site 365} \\
\hline $2-3,56-58$ & 323.56 & Miocene & 0.71 & 5.92 & 21.26 & 0.70 & 20.56 & 3.1 & 16.3 & 80.6 & 3.3 & -27.0 & -25.9 & 3.13 \\
\hline $3-1,80-82$ & 396.80 & (Reworked & 0.11 & 0.92 & 17.32 & 0.27 & 17.05 & 2.4 & 14.1 & 83.5 & 1.6 & -28.4 & -28.1 & 2.99 \\
\hline $3-1,78-80$ & 396.78 & Cretaceous) & 0.05 & 0.42 & 17.43 & 0.26 & 17.17 & 1.0 & 16.2 & 82.8 & 1.5 & -26.2 & -28.6 & - \\
\hline
\end{tabular}

Weight percent with respect to total core sample.

Weight percent with respect to total lipid fractions. Anomalously high values may result from significant content of elemental sulfur.

$c^{c}$ Weight percent with respect to total organic carbon. Anomalously high values may result from significant content of elemental sulfur.

dLeg 39.

e Insufficient sample for analysis. 
TABLE 3

n-Alkane Distribution by Carbon Number for Lipids

Extracted From Cretaceous Sediments, Sites 361 and $364^{\mathrm{a}}$

\begin{tabular}{|c|c|c|c|c|c|c|c|c|c|c|c|c|c|c|c|c|c|c|}
\hline \multirow[b]{2}{*}{ Core-Section } & \multicolumn{5}{|c|}{ Site 361} & \multirow[b]{2}{*}{$45-3$} & \multirow[b]{2}{*}{$46-3$} & \multirow[b]{2}{*}{$20-3$} & \multirow[b]{2}{*}{$21-4$} & \multirow[b]{2}{*}{$23-1$} & \multirow[b]{2}{*}{ 23-1 } & \multicolumn{3}{|c|}{ Site 364} & \multirow[b]{2}{*}{$42-2$} & \multirow[b]{2}{*}{$42-3$} & \multirow[b]{2}{*}{$43-3$} & \multirow[b]{2}{*}{$45-2$} \\
\hline & $34-2$ & $36-1$ & $37-2$ & $38-3$ & $43-4$ & & & & & & & $24-2$ & $40-2$ & $41-4$ & & & & \\
\hline \multicolumn{19}{|l|}{ Depth Below } \\
\hline Sea Floor & 1079.13 & 1096.30 & 1106.53 & 1117.62 & 1205.09 & 1241.25 & 1261.40 & 581.70 & 602.00 & 645.17 & 645.17 & 674.27 & 988.70 & 1010.03 & 1026.14 & 1027.46 & 1037.64 & 1064.80 \\
\hline 13 & & & & & & & & & & & 0.49 & & & & & & & \\
\hline 14 & & & & & & & & & & & 0.64 & & & & & & & 2.18 \\
\hline 15 & & & & & & & & & & & 0.02 & & 0.39 & & & 1.09 & & 0.74 \\
\hline 16 & & . & & & & & & 0.61 & & 4.15 & 1.36 & 1.34 & 1.52 & & 3.23 & 2.92 & & 2.69 \\
\hline 17 & & & 1.23 & 0.86 & & & & 1.81 & & 6.96 & 3.05 & 2.86 & 3.57 & & 8.04 & 8.34 & & 4.18 \\
\hline 18 & & 0.94 & 1.39 & 1.49 & & & & 2.50 & 1.38 & 1.91 & 2.27 & 4.17 & 0.30 & & 7.07 & 1.20 & 1.55 & 3.45 \\
\hline 19 & & 1.01 & 1.69 & 1.27 & & & & 2.64 & 2.11 & 4.06 & 2.97 & 3.97 & 1.77 & & 7.63 & 8.73 & 18.07 & 7.26 \\
\hline 。20 & 2.46 & 2.30 & 6.44 & 2.17 & & & & 2.52 & 2.50 & 3.12 & 2.06 & 3.03 & 2.18 & & 6.63 & 7.02 & 10.25 & 8.56 \\
\hline కัँ 21 & 1.78 & 1.95 & 2.15 & 1.67 & & & & 2.62 & 2.14 & 3.90 & 2.66 & 3.39 & 2.71 & 11.92 & 7.13 & 9.23 & 8.69 & 7.45 \\
\hline 응 22 & 1.99 & 2.33 & 1.49 & 1.77 & & & & 2.27 & 2.10 & 2.26 & 1.23 & 2.69 & 3.14 & 8.88 & 6.23 & 5.12 & 6.71 & 6.24 \\
\hline$\sum 23$ & 3.24 & 4.46 & 2.41 & 3.32 & 6.06 & & 6.85 & 5.38 & 4.20 & 4.89 & 3.40 & 4.38 & 5.40 & 13.36 & 7.56 & 10.72 & 14.52 & 13.15 \\
\hline ఏ 24 & 2.98 & 3.77 & 2.86 & 2.80 & 10.39 & & 0.00 & 2.74 & 3.08 & 2.41 & 1.58 & 2.45 & 6.60 & 9.22 & 5.97 & 4.86 & 6.32 & 6.18 \\
\hline है 25 & 6.84 & 9.92 & 4.44 & 8.03 & 6.93 & 9.53 & 13.13 & 6.73 & 5.90 & 6.37 & 4.30 & 5.37 & 9.05 & 10.96 & 6.45 & 8.36 & 12.05 & 16.30 \\
\hline อ 26 & 3.92 & 4.49 & 4.18 & 3.91 & 16.64 & 6.47 & 7.05 & 3.00 & 3.89 & 3.25 & 2.39 & 2.45 & 8.57 & 6.83 & 4.47 & 3.29 & 5.17 & 4.83 \\
\hline \pm 27 & 12.70 & 17.35 & 7.48 & 15.05 & 7.61 & 12.34 & 21.04 & 10.25 & 13.18 & 9.10 & 8.75 & 6.80 & 9.95 & 13.35 & 7.43 & 5.06 & 6.98 & 5.43 \\
\hline हू 28 & 5.61 & 5.76 & 7.80 & 5.93 & 18.44 & 6.05 & 8.43 & 3.80 & 5.20 & 4.01 & 4.43 & 2.77 & 7.65 & 4.39 & 3.54 & 2.45 & 4.12 & 2.74 \\
\hline 29 & 14.91 & 14.40 & 12.39 & 17.36 & 6.49 & 16.96 & 19.95 & 13.84 & 17.30 & 10.97 & 12.96 & 9.06 & 9.97 & 14.97 & 8.39 & 4.91 & 7.23 & 3.79 \\
\hline 30 & 8.78 & 5.03 & 8.32 & 5.48 & 16.35 & 7.37 & 5.91 & 3.99 & 4.95 & 5.24 & 5.37 & 3.36 & 5.47 & 0.00 & 2.80 & 2.23 & 3.10 & 1.68 \\
\hline 31 & 12.32 & 12.33 & 15.15 & 14.18 & 5.56 & 22.36 & 17.64 & 15.92 & 17.29 & 12.61 & 12.08 & 27.36 & 8.47 & 6.10 & 7.43 & 5.88 & 5.24 & 3.15 \\
\hline 32 & 6.60 & 3.25 & 7.01 & 3.43 & 5.53 & 7.69 & & 3.73 & 3.43 & 3.67 & 4.03 & 2.96 & 3.07 & & & 1.93 & & \\
\hline 33 & 6.19 & 5.21 & 6.52 & 6.07 & & 11.23 & & 9.96 & 7.87 & 7.07 & 18.56 & 7.77 & 4.50 & & & 3.44 & & \\
\hline 34 & 2.87 & 0.95 & 2.36 & 1.16 & & & & 1.57 & 1.28 & 2.16 & 2.33 & 1.23 & 1.03 & & & 0.75 & & \\
\hline 35 & 3.44 & 2.42 & 2.70 & 2.42 & & & & 2.35 & 2.20 & 1.52 & 2.20 & 2.59 & 1.10 & & & 0.95 & & \\
\hline 36 & 1.35 & 0.56 & 0.79 & 0.50 & & & & 0.54 & & 0.47 & 0.41 & & 2.51 & & & 0.70 & & \\
\hline 37 & 1.29 & 1.11 & 0.84 & 0.69 & & & & 0.90 & & 0.91 & 0.46 & & 0.83 & & & 0.84 & & \\
\hline 38 & 0.72 & 0.46 & & 0.41 & & & & 0.33 & & & & & 0.25 & & & & & \\
\hline
\end{tabular}

a) Normalized Weight Percent 
TABLE 4

Composition of Gas Obtained From Leg 40 DSDP Cores

\begin{tabular}{|c|c|c|c|c|c|c|c|c|c|c|c|c|c|c|c|}
\hline Core-Section & Nitrogen & Oxygen & $\begin{array}{l}\text { Carbon } \\
\text { Dioxide }\end{array}$ & Methane & Ethane & Propane & $\begin{array}{l}\text { Ratio of } \\
\text { Methane } \\
\text { to Ethane }\end{array}$ & Nitrogen & Oxygen & Argon $^{\mathrm{a}}$ & $\begin{array}{l}\text { Geologic } \\
\text { Age }\end{array}$ & $\begin{array}{l}\text { Subsurface } \\
\text { Depth (m) }\end{array}$ & Nitrogen & Methane & $\begin{array}{c}\begin{array}{c}\text { Isotopic } \\
\text { Composition } \\
\text { of Methane }\end{array} \\
\sigma C^{13}\end{array}$ \\
\hline \multicolumn{16}{|l|}{ Site 361} \\
\hline $28-6$ & 27.60 & 5.17 & 0.25 & 66.71 & 0.27 & 0.01 & 244 & 78.67 & 19.83 & 1.50 & $\begin{array}{l}\text { Lower } \\
\text { Cretaceous }\end{array}$ & 1000.5 & 763.2 & 764.3 & -66.0 \\
\hline $29-6$ (top) & 49.75 & 0.57 & 0.00 & 49.47 & 0.21 & & 235 & 98.68 & 0.00 & 1.32 & $\begin{array}{l}\text { Lower } \\
\text { Cretaceous }\end{array}$ & 1029 (top) & 1037.6 & 427.5 & -65.8 \\
\hline 29-6 (bottom) & 29.23 & 5.59 & 0.17 & 64.77 & 0.24 & 0.01 & 270 & 78.10 & 20.50 & 1.39 & $\begin{array}{l}\text { Lower } \\
\text { Cretaceous }\end{array}$ & 1029+(bot) & 842.9 & 773.8 & -66.1 \\
\hline $31-1$ & 38.10 & 7.33 & 0.30 & 54.02 & 0.25 & & 216 & 80.85 & 18.30 & 0.85 & $\begin{array}{l}\text { Lower } \\
\text { Cretaceous }\end{array}$ & 1048 & 313.5 & 184.2 & -63.1 \\
\hline $32-1$ & 42.14 & 7.82 & 0.25 & 49.42 & 0.23 & 0.13 & 215 & 81.49 & 17.33 & 1.18 & $\begin{array}{l}\text { Lower } \\
\text { Cretaceous }\end{array}$ & 1057.5 & 1088.9 & 529.0 & -55.2 \\
\hline $32-6$ & 52.85 & 10.55 & 0.18 & 36.17 & 0.15 & 0.01 & 241 & 81.05 & 17.44 & 1.50 & $\begin{array}{l}\text { Lower } \\
\text { Cretaceous }\end{array}$ & $1057.5+$ & 1227.4 & 348.0 & -64.2 \\
\hline 33-1 & 68.50 & 13.71 & 0.29 & 17.11 & 0.16 & & 107 & 77.70 & 10.80 & 1.50 & $\begin{array}{l}\text { Lower } \\
\text { Cretaceous }\end{array}$ & 1067 & 807.7 & 83.8 & -63.8 \\
\hline 34-2 & 64.35 & 13.46 & 0.17 & 21.94 & 0.07 & 0.007 & 313 & 78.66 & 20.01 & 1.20 & $\begin{array}{l}\text { Lower } \\
\text { Cretaceous }\end{array}$ & 1076.5 & 1227.2 & 172.4 & -62.1 \\
\hline $38-3$ & 51.69 & 8.19 & 0.27 & 39.62 & 0.21 & 0.02 & 187 & 80.42 & 18.23 & 1.35 & $\begin{array}{l}\text { Lower } \\
\text { Cretaceous }\end{array}$ & 1114.5 & 1175.0 & 370.3 & -59.7 \\
\hline $45-2$ & 71.65 & 14.60 & 0.23 & 12.12 & 0.05 & $\begin{array}{l}0.0006 \\
\left(\mathrm{i}-\mathrm{C}_{4} 0.0007\right. \\
\left.\mathrm{nC}_{4} 0.001\right)\end{array}$ & 242 & 89.79 & 9.18 & 1.03 & $\begin{array}{l}\text { Lower } \\
\text { Cretaceous }\end{array}$ & 1240 & 1292.5 & 91.0 & -66.1 \\
\hline \multicolumn{16}{|l|}{ Site 362} \\
\hline $8-5$ & 83.28 & 14.49 & & & & & & 78.76 & 17.93 & 3.3 & Pleistocene & 112.0 & 54.5 & - & d \\
\hline $11-6$ & 80.37 & 14.42 & 1.38 & 3.83 & & & & 79.85 & 18.43 & 1.72 & Pliocene & 140.5 & 59.63 & 1.31 & d \\
\hline $12-3$ & 58.21 & 10.42 & 3.50 & 27.87 & & & & 82.63 & 17.37 & c & Pliocene & 150. & 76.8 & 15.2 & d \\
\hline $15-3$ & 14.15 & 0.82 & 4.37 & 80.65 & & & & 93.06 & 6.94 & c & U. Miocene & 178.5 & 92.5 & 218.4 & -71.6 \\
\hline $22-3$ & 30.95 & 2.63 & 2.64 & 63.77 & & & & 90.32 & 8.38 & 1.29 & M. Miocene & 425.5 & 519.1 & 443.1 & -73.4 \\
\hline
\end{tabular}

Argon poorly resolved

Oxygen value is actually argon value.

c Argon area too small to calculate.

d Insufficient sample to determine isotopic composition. 


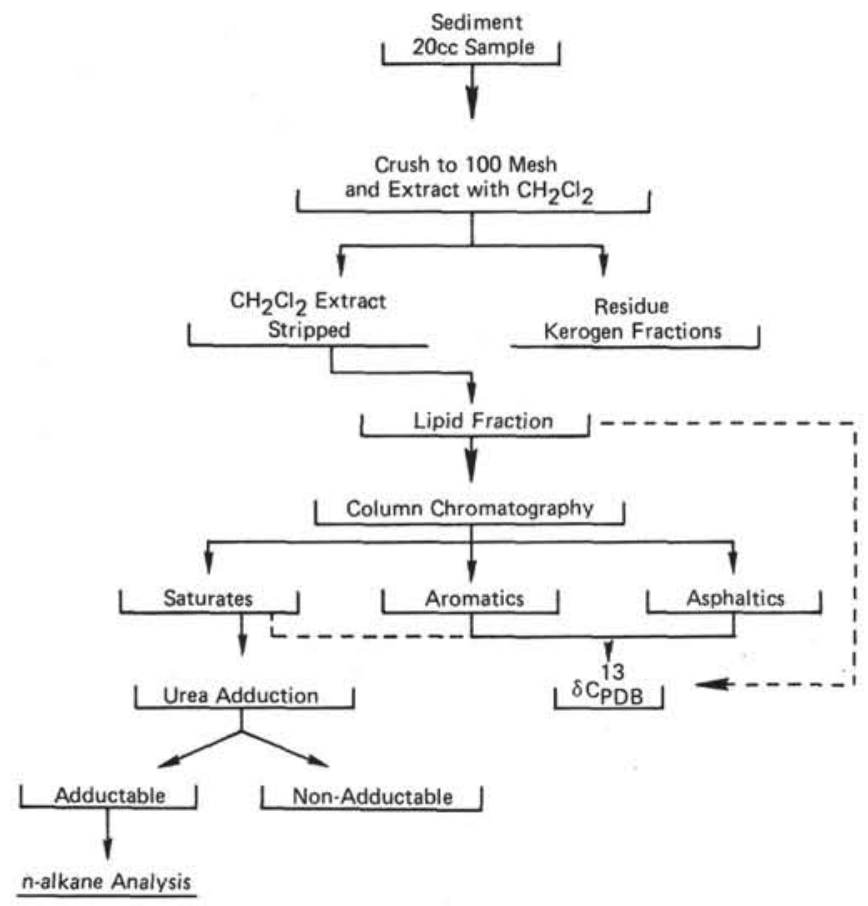

Figure 2. General laboratory procedure followed in characterizing the organic matter in sediment samples.

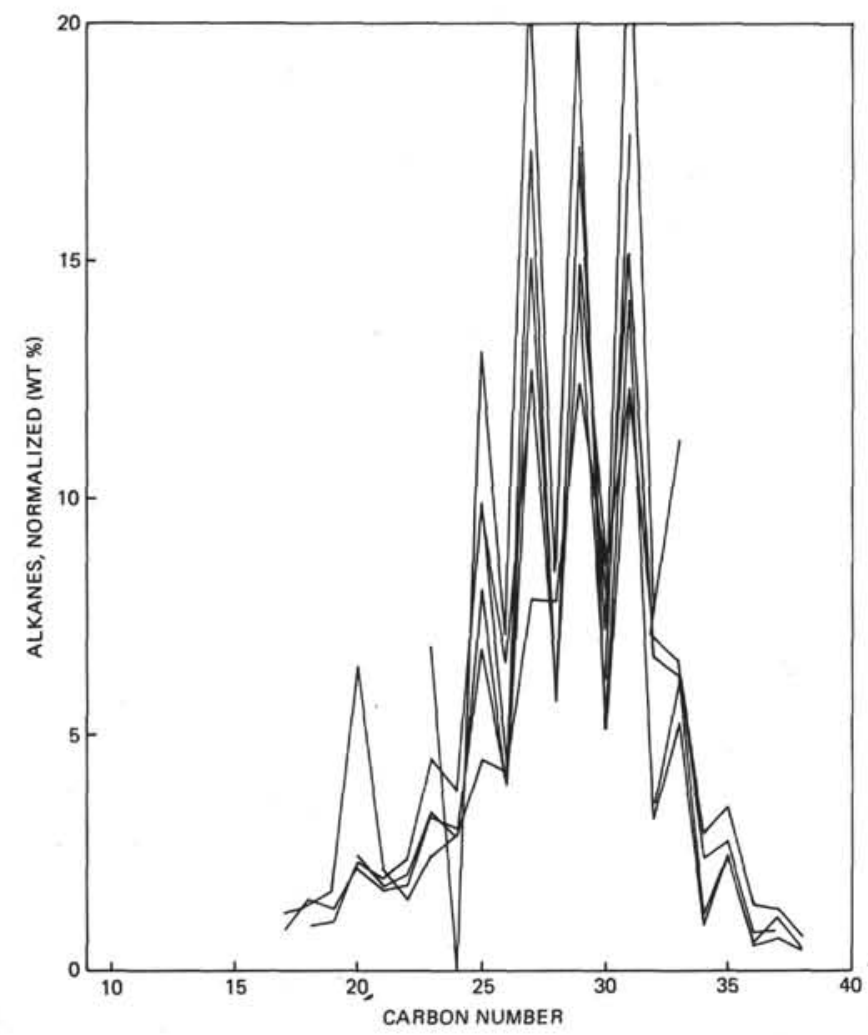

Figure 3. Plot of normalized n-alkane distribution as a function of carbon number for samples of Lower Cretaceous age recovered between 1005 and 1270 meters subbottom depth in the Cape Basin at Site 361 during Leg 40.

composition of carbon in the methane is less than -70 , which is typical of a gas derived from biogenic or a very early stage of abiogenic genesis.

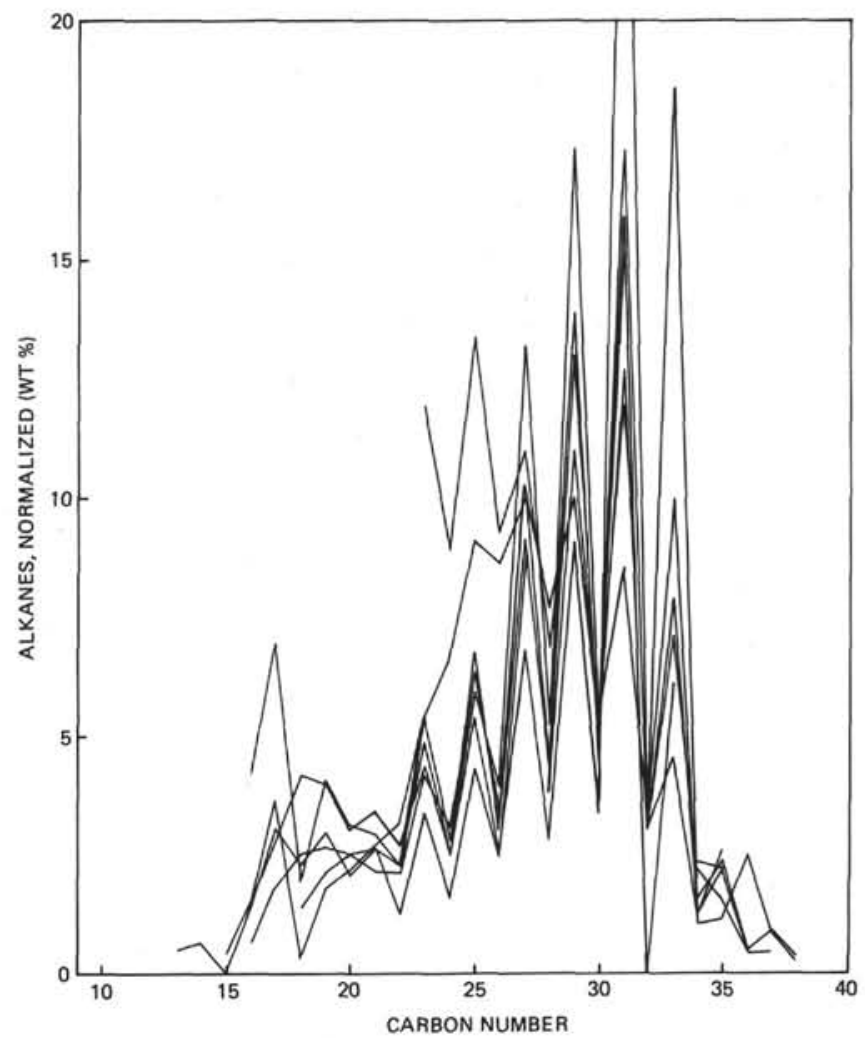

Figure 4. Plot of normalized n-alkane distribution as a function of carbon number for samples of Lower Cretaceous age recovered between 1000 and 1020 meters subbottom depth in the Angola Basin at Site 364 during Leg 40.

Organic carbon averages about $2.5 \%$ through beds of nannofossil ooze, but decreases sharply to about $0.5 \%$ or less in the underlying chalk. Isotopic composition of the lipid extract is typical of that deposited in openmarine environment with little or no contribution from a terrestrial source.

Site 363 was selected to permit sampling in a single hole of stratigraphic overlap with Holes 362 and $362 \mathrm{~A}$, and a continuation into beds of Lower Cretaceous age. The hole was terminated at a subsea depth of 715 meters because of bit problems. The section recovered extends in age from upper Miocene to lower Aptian, with two prominent erosional gaps-one between the Recent and upper Miocene, the other between Coniacian and the uppermost Albian. The younger beds primarily consist of biogenic oozes, and in descending order, chalks, marl, and limestone. The beds of Albian age are largely calcareous, but characterized by intercalations of dark shaly to nearly sapropelic layers. The lowermost beds of Aptian age consist of shallow water limestone interlayered with calcarenites.

Only representative beds of Cretaceous age were sampled at Site 363. Although the argillaceous parts of the generally calcareous section were too low in organic content to permit full characterization of carbon, the isotopic composition of the total carbon extract falls within the range expected of an open-marine environment. 


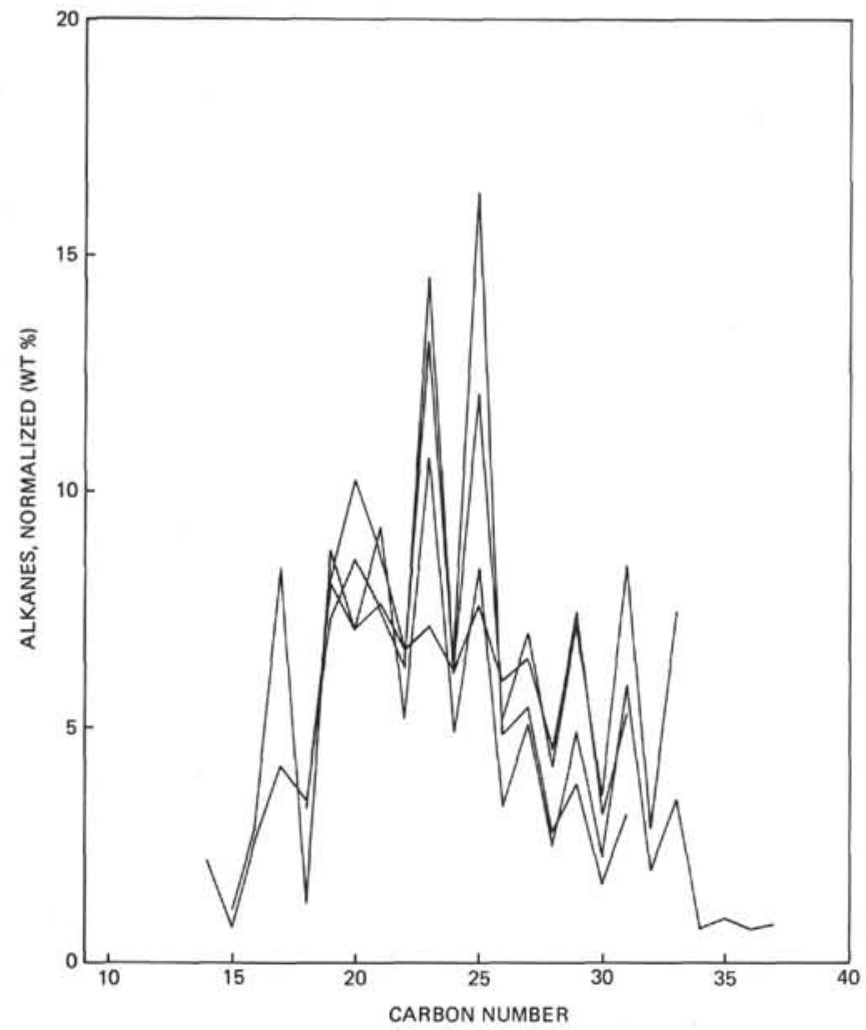

Figure 5. Plot of normalized n-alkane distribution as a function of carbon number for samples of Lower Cretaceous age recovered between 1020 and 1065 meters in the Angola Basin at Site 364 during Leg 40. Note the shift from the heavier hydrocarbons shown in Figure 4 to the lighter fraction shown above. Although still immature, this indicates that genesis of petroleum is beginning to occur with depth.

\section{Angola Basin}

Sites 364 and 365 were drilled in the Angola Basin to subbottom depths of 1086 and 687 meters, respectively. Sediments penetrated at Site 364 ranged in age from upper Eocene down through 727 meters of Cretaceous ending in beds of Aptian age. This section began in calcareous mud and clay, and was drilled down through marly nanno ooze, pelagic clay, nanno chalk, and marly chalk of Upper Cretaceous age and into intercalated mudstones, dark sapropelic appearing shale, marly limestone, and dolomite of Lower Cretaceous age.

Site 365 was selected as a last attempt to sample the lowest Cretaceous beds by lowering the drill string into a submarine cleft. The beds penetrated, however, are believed by some paleontologists to be of Miocene age with only reworked sediments of Cretaceous age. In order to maximize the rate of penetration, only seven cores were recovered. These largely consist of mudstone and nearly sapropelic layers similar to those of the Lower Cretaceous section recovered at Site 364.

Most of the cores that were recovered at Site 364 were analyzed for carbonate carbon versus organic carbon. Reducing conditions appear to have persisted through Aptian time and, unlike the Cape Basin,

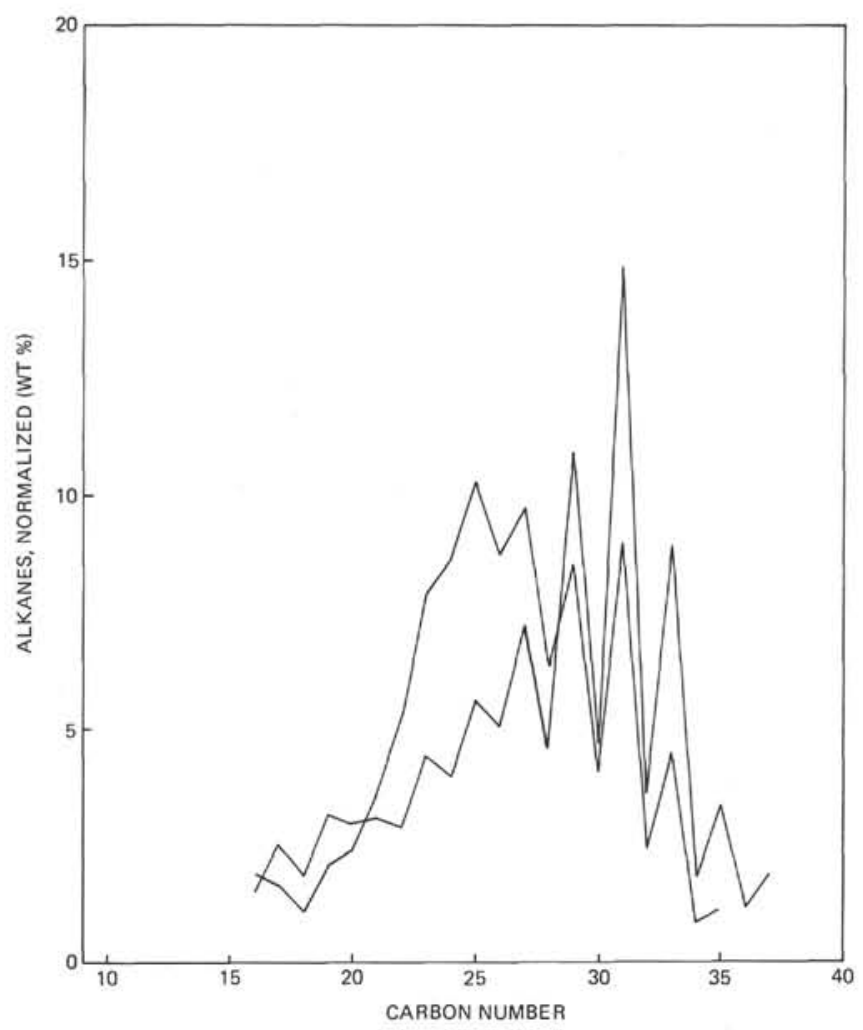

Figure 6. Plot of normalized n-alkane distribution as a function of carbon number for samples of Lower Cretaceous age recovered from the western part of the Angola Basin at Site 356 on Leg 39. Note the similarity of peak positions with those shown in Figure 4 for similar age samples recovered from the Angola Basin, at Site 364, Leg 40.

recurred in late Albian and again in Santonian time. The organic content, however, is significantly higher in the Lower Cretaceous sediments. As with the Aptian carbonaceous beds at Site 361 in the Cape Basin, many of the samples from Lower Cretaceous beds contain sufficient organic material to qualify as source beds of petroleum. Although the OEP values indicate that organic diagenesis is in an early stage, a shift from heavy hydrocarbons to lighter components with depth suggests that genesis is proceeding slowly with depth.

The carbon isotopic values of the lipid fraction suggest that there has been a sporadic introduction of terrigenous material to this part of the Angola Basin during Aptian and lower Albian time.

Carbon analyses also were performed on 10 samples of organic-rich sediments of Cretaceous age from Site 356 which was drilled off the continental rise of South America in the Sao Paulo Plateau during Leg 39. Two of these contained sufficient amount of organic material to permit geochemical characterization of the lipid fraction. These samples were found to be remarkably similar to those recovered at Site 364 , in lipid content, degree of genesis, and isotopic composition.

\section{SUMMARY AND CONCLUSIONS}

Petroleum genesis is evident at a depth of about 1000 meters in the Lower Cretaceous strata penetrated at 
sites in both the Cape and Angola basins. The first products of this thermal diagenesis include gas which consists primarily of isotopically light methane with an ethane component and small quantities of higher homologs. Moreover, the carbonaceous beds in both areas contain in situ lipid (oil) which can be distinguished and treated as saturates, aromatics, and asphaltics. Samples of time-lithologic equivalents that were recovered from Site 356 on the Sao Paulo Plateau during Leg 39 also were analyzed and found to be geochemically similar. Isotopic compositions of carbon from Lower Cretaceous beds both in the Cape and Angola basins indicate a marine environment that received a significant contribution of terrigenous carbon. During that time these environments were either located near the ancient strand line or could be reached by turbidity flows. In contrast there was little influx of land-derived material to these areas after the Lower Cretaceous; Tertiary sedimentation was largely pelagic. Nevertheless, reducing conditions appear to have ceased in Cape Basin by the end of Aptian time, while they persisted into Santonian time north of the Walvis Ridge. Altogether these findings suggest that lithologic equivalents of the Lower Cretaceous sediments cored at Sites 361 and 364 which are known to thicken and became more deeply buried toward the present continental shelf of southwest Africa in both basins (Emery et al., 1975) may have been preserved at sufficient depth to have generated significant quantities of oil.

\section{ACKNOWLEDGMENT}

The author expresses appreciation to W.J. Hines, C.G. Long, and M.E. Smith who each helped in assembling and testing geochemical equipment for the leg; to C.R. Bennett who supervised the mass spectrometric analyses; to K.S.
Schorno who conducted the gas-liquid chromatography; to T.V. Iorns who supervised the determination of carbonate versus organic carbon content; to J.G. Erdman and D.A. Morris who both offered helpful suggestions throughout this study; and to members of the JOIDES Organic Geochemistry Panel, who made my participation in Leg 40 possible.

\section{REFERENCES}

Bray, E.E. and Evans, E.D.; 1961. Distribution of n-paraffins as a clue to recognition of source beds: Geochim. Cosmochim. Acta, v. 22, p. 2-15.

Craig, H., 1953. The geochemistry of the stable carbon isotopes: Geochim. Cosmochim. Acta, v. 3, p. 53-92.

Emery, K.O., Uchupi, E., Bowin, C.O., Phillips, J., and Simpson, E.S.W., 1975. The continental margin off western Africa: Cape St. Francis (South Africa) to Walvis Ridge (Southwest Africa): Am. Assoc. Petrol. Geol. Bull., v. 59 , p. $3-59$.

Erdman, J.G., 1975a. Geochemical formation of oil. In Fisher, A.G. and Judson, S. (Eds.), Petroleum and global tectonics, Princeton (Princeton University Press), p. 225248.

1975b. Relations controlling oil and gas generation in sedimentary basins, Paper 1, Panel Discussion 3, Proceedings of 9th World Petroleum Congress, Tokyo, p. $139-148$

Philippi, G.T., 1956. Identification of oil source beds by chemical means: Internatl. Geol. Congr., 20th Mexico, Section III, p. 25-38.

Rosenfeld, W.D. and Silverman, S.R., 1959. Carbon isotope fractionation in bacterial production of methane: Science, v. 130 , p. 1658.

Scalan, R.S. and Smith, J.E., 1970. An improved measure of the Odd-Even Predominance in the normal alkanes of sediment extracts and petroleum; Geochim. Cosmochim. Acta, v. 34, p. 611-630.

Smith, J.E., Erdman, J.G., and Morris, D.A., 1971. Migration, accumulation and retention of petroleum in the earth: 8th World Petrol. Congr. Proc., v. 2, p. 13-26. 